\title{
The WorldWide Antimalarial Resistance Network Clinical Trials Publication Library: A Live, Open-Access Database of Plasmodium Treatment Efficacy Trials
}

\author{
Junko Takata, ${ }^{1,2}$ Paul Sondo, ${ }^{1,2,3}$ Georgina S. Humphreys, ${ }^{1,2}$ Rebekah Burrow, ${ }^{1,2,4}$ Brittany Maguire, ${ }^{2,4}$ Mohammad S. Hossain, ${ }^{1,2,5}$ \\ Debashish Das, ${ }^{1,2,4}$ Robert J. Commons, ${ }^{1,2,6}$ Ric N. Price, ${ }^{1,2,4,6,7}$ and Philippe J. Guerin ${ }^{1,2,4 *}$ \\ ${ }^{1}$ WorldWide Antimalarial Resistance Network (WWARN), Oxford, United Kingdom; ${ }^{2}$ Infectious Diseases Data Observatory (IDDO), Oxford, United \\ Kingdom; ${ }^{3}$ Institut de Recherche en Sciences de la Santé (IRSS)/Clinical Research Unit of Nanoro (CRUN), Nanoro, Burkina Faso; ${ }^{4}$ Centre for \\ Tropical Medicine and Global Health, Nuffield Department of Clinical Medicine, University of Oxford, Oxford, United Kingdom; ${ }^{5}$ International Centre \\ for Diarrhoeal Disease Research, Bangladesh (icddr,b), Dhaka, Bangladesh; ${ }^{6}$ Global Health Division, Menzies School of Health Research, Charles \\ Darwin University, Darwin, Australia; ${ }^{7}$ Mahidol-Oxford Tropical Medicine Research Unit (MORU), Faculty of Tropical Medicine, Mahidol University,
} Bangkok, Thailand

\begin{abstract}
Parasite resistance to antimalarial drugs poses a serious threat to malaria control. The WorldWide Antimalarial Resistance Network (WWARN) aims to provide a collaborative platform to support the global malaria research effort. Here, we describe the "WWARN clinical trials publication library," an open-access, up-to-date resource to streamline the synthesis of antimalarial safety and efficacy data. A series of iteratively refined database searches were conducted to identify prospective clinical trials assessing antimalarial drug efficacy with at least 28 days of follow-up. Of approximately 45,000 articles screened, 1,221 trials published between 1946 and 2018 were identified, representing 2,339 treatment arms and 323,819 patients. In trials from endemic locations, $75.7 \%(787 / 1,040)$ recruited patients with Plasmodium falciparum, $17.0 \%(177 / 1,040)$ Plasmodium vivax, $6.9 \%(72 / 1,040)$ both, and $0.4 \%(4 / 1,040)$ other Plasmodium species; $57.2 \%(585 / 1,022)$ of trials included under-fives and $5.3 \%(55 / 1,036)$ included pregnant women. In Africa, there has been a marked increase in both $P$. falciparum and $P$. vivax studies over the last two decades. The WHOrecommended artemisinin-based combination therapies alone or with a gametocidal drug were assessed in $39.5 \%$ $(705 / 1,783)$ of $P$. falciparum treatment arms and $10.5 \%$ (45/429) of $P$. vivax arms, increasing to $78.0 \%(266 / 341)$ and $22.9 \%(27 / 118)$, respectively, in the last five years. The library is a comprehensive, open-access tool that can be used by the malaria community to explore the collective knowledge on antimalarial efficacy (available at https:// www.wwarn.org/tools-resources/literature-reviews/wwarn-clinical-trials-publication-library). It is the first of its kind in the field of global infectious diseases, and lessons learnt in its creation can be adapted to other infectious diseases.
\end{abstract}

\section{INTRODUCTION}

One of the greatest challenges facing malaria control is the ability of Plasmodium species to adapt to selective drug pressure. Since World War II, resistance has emerged and spread to all antimalarials that were used en masse in malariaendemic countries. The development of artemisinin-based combination therapies (ACTs) was an attempt to prevent or slow down antimalarial resistance, but although they remain efficacious first-line treatments in most of the malariaendemic world, resistance to the artemisinin derivatives and their partner drugs is now emerging. ${ }^{1}$ Synthesizing the available evidence on antimalarial efficacy is essential to understand the drivers of resistance, ensure the optimal use of available treatment options, and facilitate timely and appropriate decision-making by policy-makers.

The WorldWide Antimalarial Resistance Network (WWARN) was established in 2009, with the aim to provide innovative tools and a collaborative data platform to facilitate the optimal use of antimalarial drugs and support the global research effort toward malaria elimination. One such tool is the "WWARN clinical trials publication library," a comprehensive, systematically constructed database of published antimalarial efficacy trials. The library is actively maintained with periodic updates and can be rapidly searched for relevant studies with pre-extracted data, thus expediting the process of evidence identification and synthesis. To date, this repository has been

*Address correspondence to Philippe J. Guerin, WorldWide Antimalarial Resistance Network (WWARN), Centre for Tropical Medicine \& Global Health, New Richards Building, Old Road Campus, Roosevelt Drive, Oxford, OX3 7LG. E-mail: philippe.guerin@wwarn.org used to undertake a series of systematic reviews and individual patient data meta-analyses within the WWARN, several of which have resulted in the optimization of antimalarial dosing regimens. ${ }^{2-9}$ Since 2011 , the library has been freely available on the WWARN website as an open-access tool for the wider malaria community (https://www.wwarn.org/toolsresources/literature-reviews/wwarn-clinical-trials-publicationlibrary). ${ }^{10}$

Since its inception, the project has evolved, requiring modification of underlying methods and repository format. This article describes the open-source library and the data contained within it, articulates the lessons learnt in its creation, and describes its utility as a resource-efficient model that avoids substantial duplication of effort when tracking antimalarial efficacy trials. The application of this model in other diseases is also explored.

\section{MATERIALS AND METHODS}

Literature search and study selection. Between 2011 and July 2018, a series of searches were conducted in PubMed, Embase, Web of Science Core Collection, and Central databases. Publications were screened by title, abstract, and full text as required. Since 2011, there have been slight variations in the methods used for the searches, screening, and data extraction. These variations include the selection of available databases used in the periodic searches, the time period during which trials were published, the number of reviewers undertaking the research, and the inclusion and exclusion criteria. These variations can be categorized into three main search iterations (documented in Supplemental Tables 1 and 2). The different inclusion and exclusion criteria were then 
rationalized to the criteria summarized in Table 1 . The final library presented in this report is restricted to prospective studies assessing antimalarial drug efficacy to any humaninfecting Plasmodium species, with a follow-up period of at least 28 days in accordance with the current WHO guidelines. ${ }^{11}$ A summary of the searches is presented in Figure 1.

Data extraction. Variables relating to study design, patient demographics, Plasmodium species, antimalarial treatment, and study site were extracted. Initially, data were entered into Microsoft Excel and then subsequently revised using REDCap (Vanderbilt University, Nashville, TN), an electronic data capture tool hosted at the University of Oxford. ${ }^{12,13}$ For nonEnglish language publications, data were extracted fully by a speaker of that language or partially extracted as much as possible by a non-native speaker using translation tools. Data for each treatment arm and study site in a publication were extracted separately and according to species if a publication assessed both Plasmodium falciparum and Plasmodium vivax. Each study may, therefore, have multiple associated treatments and study sites. Time measures were extracted to the closest year (i.e., year of publication and start and end year of recruitment). The full list and definitions of extracted variables can be found in Supplemental Table 3.

Data categorization. Countries were classified into regions and subregions as per the United Nations designation of areas and regions. ${ }^{14}$ Treatments were categorized as follows:

1. Artemisinin-based combination therapies currently recommended by the WHO (artemether-lumefantrine, artesunate-mefloquine, dihydroartemisinin-piperaquine, artesunate-amodiaquine, and artesunate-sulfadoxinepyrimethamine) or registered to stringent regulatory authorities (artesunate-pyronaridine): all either alone or in combination with gametocidal drugs (primaquine or tafenoquine),

2. any other artemisinin-based therapies,

3. chloroquine,

4. chloroquine in combination with other drugs,

5. quinine,

6. quinine in combination with other drugs, and

7. other.

A full list of all treatment regimens and their classifications can be found in Supplemental Table 4.

Data analysis. The primary unit of analysis reported in the Results section is "publication." The term "study site" is used for each longitude/latitude point on the maps which include all of the individual study sites in one country. The term "study location" is used to infer the country or countries in which a study was conducted rather than the individual study sites. The term "treatment arm" is the unit of analysis for exploring trends in treatment. Reported proportions are calculated using the number of relevant data points available for extraction as the denominator, with the denominators varying according to the number of missing data points. When analyzing time trends, the year 2018 was not included as data were available for only part of the year. Data analysis was conducted in RStudio (version 1.1.463).

\section{RESULTS}

Approximately 45,000 papers were screened to identify a total of 1,221 clinical trials published since 1946 (Figure 1). Overall, 323,819 patients were recruited in 2,339 treatment arms in the study period. Thirty-six studies investigated imported malaria from returning travelers.

Descriptive characteristics. Of the 1,185 studies of nonimported malaria, 1,179 $(99.5 \%)$ recorded location data, of which 524 (44.4\%) were conducted in Africa, 523 (44.4\%) in Asia, and 97 (8.2\%) in South and Central America (Table 2). The species of infection was recorded in 1,040 (87.8\%) of studies, with $P$. falciparum assessed in $75.7 \%$ (787), $P$. vivax in $17.0 \%$ (177), and both species in 6.9\% (72) (Table 3). The remaining four $(0.4 \%)$ studies investigated only other species, that is, Plasmodium malariae and Plasmodium ovale (together in two studies) and Plasmodium knowlesi (two studies). A further 19 studies also assessed $P$. malariae, $P$. ovale, and/or $P$. knowlesi alongside $P$. falciparum and/or $P$. vivax. The majority of studies assessing $P$. falciparum were in Africa (54.1\%), whereas those assessing $P$. vivax were mostly undertaken in Asia (67.8\%).

One thousand thirty-six (87.4\%) studies recorded information on pregnancy status, of which 55 (5.3\%) recruited pregnant women; 52 (94.5\%) studies were in patients with $P$. falciparum and $36(65.5 \%)$ studies were conducted in Africa. Age information was recorded in 1,022 (86.2\%) studies. Children younger than 5 years were included in 585 (57.2\%) studies, of which $456(78.0 \%)$ were $P$. falciparum studies and $83(14.2 \%)$ P. vivax; 342 (58.5\%) studies were conducted in Africa and 189 (32.3\%) in Asia. One hundred thirty-three (13.0\%) studies were restricted to children younger than 5 years, all but eight of them published after 2000; 124 (93.2\%) studies investigated patients with $P$. falciparum which were all undertaken in Africa (Supplemental Table 6a). Although the

TABLE 1

Inclusion and exclusion criteria used for the final library

\begin{tabular}{|c|c|}
\hline Inclusion criteria & Exclusion criteria \\
\hline Human-infecting Plasmodium species (including mixed infections) & Animal studies \\
\hline Prospective studies & Prevention or prophylaxis studies \\
\hline Assessing efficacy of any antimalarial drug & $\begin{array}{l}\text { Intermittent preventive treatment studies and intermittent } \\
\text { screening and treatment studies in pregnancy }\end{array}$ \\
\hline Follow-up of at least 28 days & Mass drug administration \\
\hline $\begin{array}{l}\text { From database inception until the date of most recent search } \\
\text { (currently July } 27,2018 \text { ) }\end{array}$ & Studies including only severe malaria \\
\hline \multirow[t]{5}{*}{ Any language } & Herbal medicine \\
\hline & Reviews or pooled analyses \\
\hline & Case reports \\
\hline & Conference abstracts \\
\hline & Asymptomatic/induced malaria studies \\
\hline
\end{tabular}




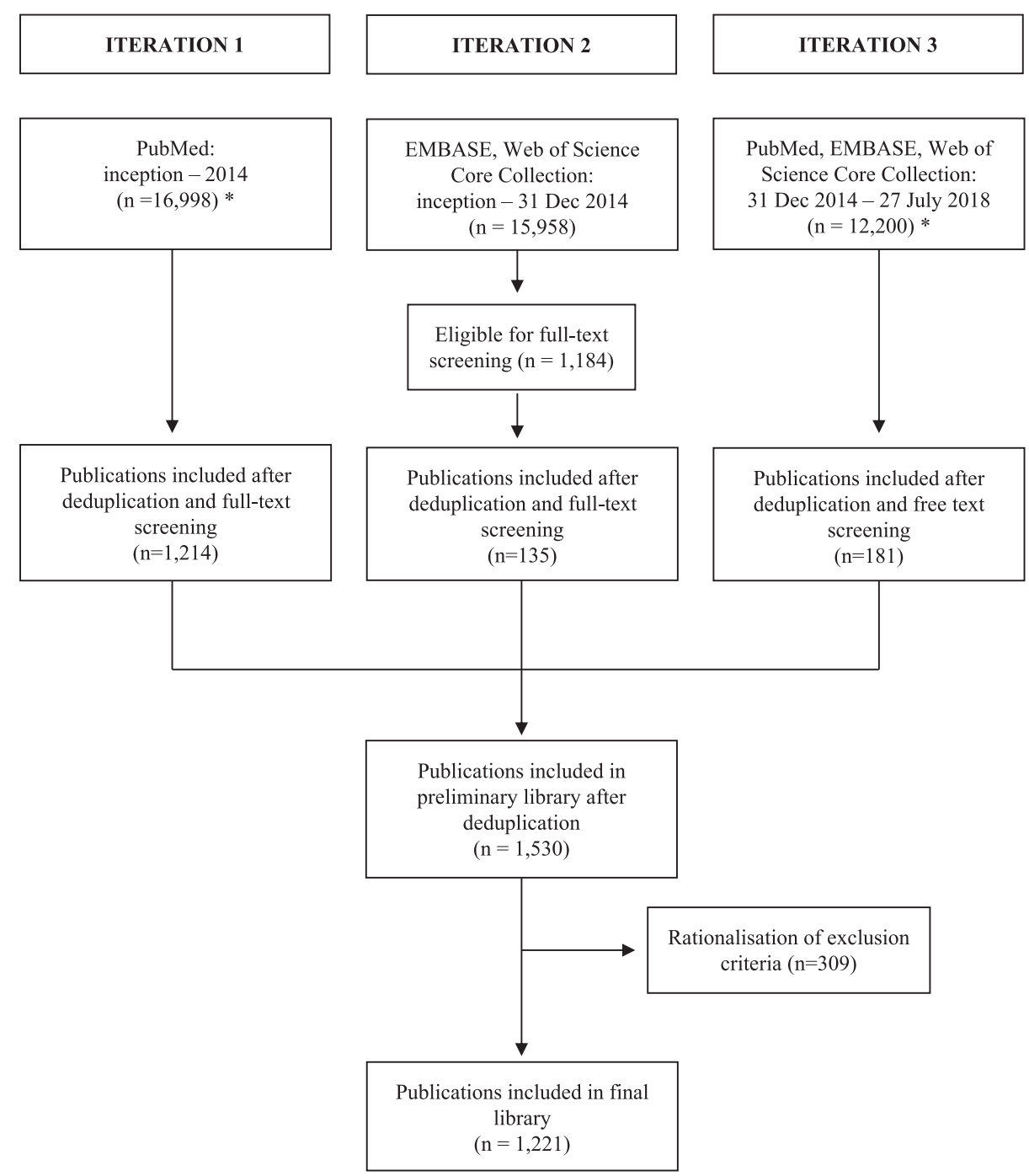

FIGURE 1. Summary of searches. Iteration numbers correspond to the iterations as described in Supplemental Table 1. For iterations 1 and 3 , the numbers denoted by $\left(^{*}\right)$ are representative figures computed retrospectively, as summarized in Supplemental Table 5.

$124 P$. falciparum studies restricted to children younger than 5 years constitute only $16.0 \%(124 / 774)$ of all $P$. falciparum trials with available age information, these studies recruited $22.7 \%$ $(51,039 / 224,930)$ of all $P$. falciparum patients and $27.5 \%$ $(34,766 / 126,532)$ of all $P$. falciparum patients treated with a WHO-recommended ACT.

Of 1,039 studies with information on study design, 568 $(54.7 \%)$ of studies were randomized, of which $95(16.7 \%)$ were at least single blinded. The median duration for study recruitment to the nearest year was 1 year (range: < 1-15 years), and the median time from the end of participant recruitment to publication to the nearest year was 2 years (range: $<1-13$ years).

Temporal trends. The number of antimalarial efficacy trials published per year has increased year on year, until a peak in 2009 for $P$. falciparum and in 2015 for $P$. vivax (Figure 2). Overall, $69.5 \%$ (547/787) of $P$. falciparum studies were published on or after 2000 , with this increase particularly marked in Africa. There has also been an increase in $P$. vivax studies over time, with $81.9 \%(145 / 177)$ published on or after 2000 , including 15 P. vivax studies conducted in Africa.
The total number of patients recruited per year was similar over time and region in $P$. falciparum studies, although the trend fluctuated for $P$. vivax because of a few very large trials (Figure 3). The median number of patients recruited per treatment arm was 77 (interquartile range (IQR): 40-151) for $P$. falciparum treatment arms and 63 (IQR: 28-125) for $P$. vivax.

Spatial trends. Of 1,179 studies with location data, 55 $(4.7 \%)$ studies recruited patients from multiple countries, 51 $(92.7 \%)$ of which were conducted after the year 2000 . Overall, there were 1,340 study locations across 82 unique countries, of which $P$. falciparum was studied in 931 locations, $P$. vivax in 185 , both species in 72, and only other species in 4 . For 148 locations, the species were not specified in the text.

The greatest number of $931 P$. falciparum studies was undertaken in Thailand (140, 15.0\%), followed by Nigeria (72, $7.7 \%)$, India (55, 5.9\%), and Kenya (41, 4.4\%) (Figure 4). However, in the last 5 years, since 2013, the proportion of studies enrolling patients in Thailand has fallen to $2.6 \%(6 / 232)$ (Supplemental Figure 1), with an associated increase in studies undertaken in Africa. The greatest number of 185 $P$. vivax studies was undertaken in Thailand $(35,18.9 \%)$ and 
TABLE 2

Geographical and language distribution of studies

\begin{tabular}{lrr}
\hline & Number & $\%$ \\
\hline Region ( $n=1,179)$ & & \\
Africa & 524 & 44.4 \\
Northern Africa & 28 & 2.4 \\
Eastern Africa & 182 & 15.4 \\
Western Africa & 193 & 16.4 \\
Middle Africa & 84 & 7.1 \\
Southern Africa & 5 & 0.4 \\
Multi-Africa region & 32 & 2.7 \\
Asia & 523 & 44.4 \\
South Asia & 143 & 12.1 \\
Southeast Asia & 324 & 27.5 \\
East Asia & 40 & 3.4 \\
Western Asia & 7 & 0.6 \\
Multi-Asia region & 9 & 0.8 \\
South and Central America & 97 & 8.2 \\
South America & 88 & 7.5 \\
Central America and Caribbean & 8 & 0.7 \\
Multi-America region & 1 & 0.1 \\
Oceania (Melanesia) & 22 & 1.9 \\
Multi-region & 13 & 1.1 \\
Language ( $n=1,185)$ & & \\
English & 1,072 & 90.5 \\
French & 57 & 4.8 \\
Chinese & 27 & 2.3 \\
Portuguese & 11 & 0.9 \\
Spanish & 10 & 0.8 \\
Other & 8 & 0.7 \\
\hline Percentages are derived using the number of studies with available data for that variable as \\
(1) & & \\
\hline
\end{tabular}

the denominator, denoted by $n$.

India (31, 16.8\%); in the last 5 years, there has been a rising proportion of studies from Brazil and Ethiopia.

Figure 5 shows the distribution of individual study sites. The most common $P$. falciparum study sites overall were Bangkok, Thailand ( $n=67)$, Ibadan, Nigeria $(n=44)$, Mae Sot, Thailand ( $n=34)$, and Lambaréné, Gabon $(n=29)$, whereas the most common $P$. vivax study sites were in Bangkok $(n=20)$ and Mae Sot $(n=8)$. The progression in the distribution of study sites is shown in Supplemental Figure 2. Before the 2000s, most studies were focused in Southeast Asia and India. Since the 2000s, there has been a surge in the number of study sites in Africa; in the last 5 years, this has included sites such as Tororo, Uganda $(n=8)$, Kinshasa, Democratic Republic of the Congo $(n=7)$, or Nanoro, Nouna, and Ouagadougou in Burkina Faso
( $n=6$ each). There has also been an increase in $P$. vivax studies in South America and Africa, mainly in Ethiopia.

Treatments. Overall, 2,264 treatment arms were assessed, enrolling 319,167 patients. A total of 236,941 patients with $P$. falciparum were enrolled into 1,783 treatment arms. Artemisinin-based therapies were assessed in 52.9\% (944/ $1,783)$ of arms, of which $39.5 \%(705 / 1,783)$ included a WHOrecommended ACT, alone or with a gametocidal drug. The number of treatment arms including an ACT has increased substantially over time (Figure 6), particularly in Africa (Supplemental Figure 3). The most commonly studied regimen was artemether-lumefantrine, which was assessed in $14.0 \%$ $(250 / 1,783)$ of treatment arms and $19.5 \%(46,280 / 236,941)$ of patients (Figures 7 and 8 ). In the last 5 years, since 2013, the number of treatment arms assessing artemisinin-based therapies and WHO-recommended ACTs rose to $88.6 \%(302 / 341)$ and $78.0 \%$ (266/341), respectively. There has also been a substantial reduction in the proportion of treatment arms testing non-artemisinin drugs, such as sulfadoxine-pyrimethamine or chloroquine (Supplemental Figure 4).

A total of 78,377 patients with $P$. vivax were enrolled into 429 treatment arms. The predominant antimalarial regimen used was chloroquine, which was assessed either alone or in combination with another drug (excluding artemisinin derivatives) in $64.6 \%(277 / 429)$ of treatment arms. Artemisininbased therapies were assessed in $85(19.8 \%)$ treatment arms and WHO-recommended ACTs in $45(10.5 \%)$; in the last 5 years, this proportion rose to $29.7 \%(35 / 118)$ and $22.9 \%$ (27/118), respectively. Of the 45 treatment arms testing a WHO-recommended ACT, $37.8 \%$ (17) were in combination with primaquine.

Nineteen treatment arms assessed 352 patients with mixed $P$. falciparum and $P$. vivax infection (with one arm assessing mixed $P$. falciparum, $P$. vivax, and $P$. malariae infection), of which $10(52.6 \%)$ treatment arms used artemisinin-based therapies and $6(31.6 \%)$ used chloroquine combinations. Sixteen treatment arms assessed 695 patients with other Plasmodium species (with one arm assessing mixed $P$. malariae and $P$. vivax infection); chloroquine combinations $(62.5 \%, 10 /$ 16) were most commonly used, whereas artemisinin-based therapies were used in $5(31.2 \%)$ treatment arms. The remaining 17 treatment arms assessing 2,802 patients did not specify the species in the text.

TABLE 3

Participant demographics and species assessed, stratified by region

\begin{tabular}{|c|c|c|c|c|c|c|c|c|c|c|c|c|}
\hline \multirow[b]{2}{*}{ Age of participants $(n=1,022)^{\star}$ (years } & \multicolumn{2}{|c|}{ Asia } & \multicolumn{2}{|c|}{ Africa } & \multicolumn{2}{|c|}{$\begin{array}{c}\text { South and } \\
\text { Central America }\end{array}$} & \multicolumn{2}{|c|}{ Oceania } & \multicolumn{2}{|c|}{ Multi-region } & \multicolumn{2}{|c|}{ Total } \\
\hline & & & & & & & & & & & & \\
\hline$<5$ only & 2 & $1.5 \%$ & 125 & $94.0 \%$ & 0 & $0.0 \%$ & 6 & $4.5 \%$ & 0 & $0.0 \%$ & 133 & $13.0 \%$ \\
\hline $5-15$ only & 2 & $14.3 \%$ & 9 & $64.3 \%$ & 0 & $0.0 \%$ & 3 & $21.4 \%$ & 0 & $0.0 \%$ & 14 & $1.4 \%$ \\
\hline$>15$ only & 165 & $67.1 \%$ & 55 & $22.4 \%$ & 22 & $8.9 \%$ & 2 & $0.8 \%$ & 2 & $0.8 \%$ & 246 & $24.1 \%$ \\
\hline Multiple age categories & 299 & $47.5 \%$ & 249 & $39.6 \%$ & 61 & $9.7 \%$ & 9 & $1.4 \%$ & 11 & $1.8 \%$ & 629 & $61.5 \%$ \\
\hline Included pregnancy $(n=1,036)$ & 18 & $32.7 \%$ & 36 & $65.5 \%$ & 0 & $0.0 \%$ & 1 & $1.8 \%$ & 0 & $0.0 \%$ & 55 & $5.3 \%$ \\
\hline \multicolumn{13}{|l|}{ Species $(n=1,040)$} \\
\hline P. falciparum $\dagger$ & 303 & $38.5 \%$ & 426 & $54.1 \%$ & 40 & $5.1 \%$ & 6 & $0.8 \%$ & 12 & $1.5 \%$ & 787 & $75.7 \%$ \\
\hline P. vivax‡ & 120 & $67.8 \%$ & 15 & $8.5 \%$ & 37 & $20.9 \%$ & 4 & $2.3 \%$ & 1 & $0.6 \%$ & 177 & $17.0 \%$ \\
\hline Both§ & 51 & $70.8 \%$ & 3 & $4.2 \%$ & 8 & $11.1 \%$ & 10 & $13.9 \%$ & 0 & $0.0 \%$ & 72 & $6.9 \%$ \\
\hline Other species & 3 & $75.0 \%$ & 1 & $25.0 \%$ & 0 & $0.0 \%$ & 0 & $0.0 \%$ & 0 & $0.0 \%$ & 4 & $0.4 \%$ \\
\hline
\end{tabular}

$P$. falciparum $=$ Plasmodium falciparum; $P$. knowlesi $=$ Plasmodium knowlesi; $P$. malariae $=$ Plasmodium malariae; $P$. ovale $=$ Plasmodium ovale; $P$. vivax $=$ Plasmodium vivax. Percentages are expressed as a proportion of total studies for each row. Percentages in the final column are derived using the number of studies with available data for that variable as the denominator, denoted by $n$.

*These are mutually exclusive categories, that is, showing studies that were restricted to each age category.

† Includes seven studies that also assessed patients with $P$. malariae and/or $P$. ovale.

$\ddagger$ Includes one study that also assessed patients with $P$. knowlesi.

§Includes 11 studies that also assessed patients with $P$. malariae, $P$. ovale, and/or $P$. knowlesi. 


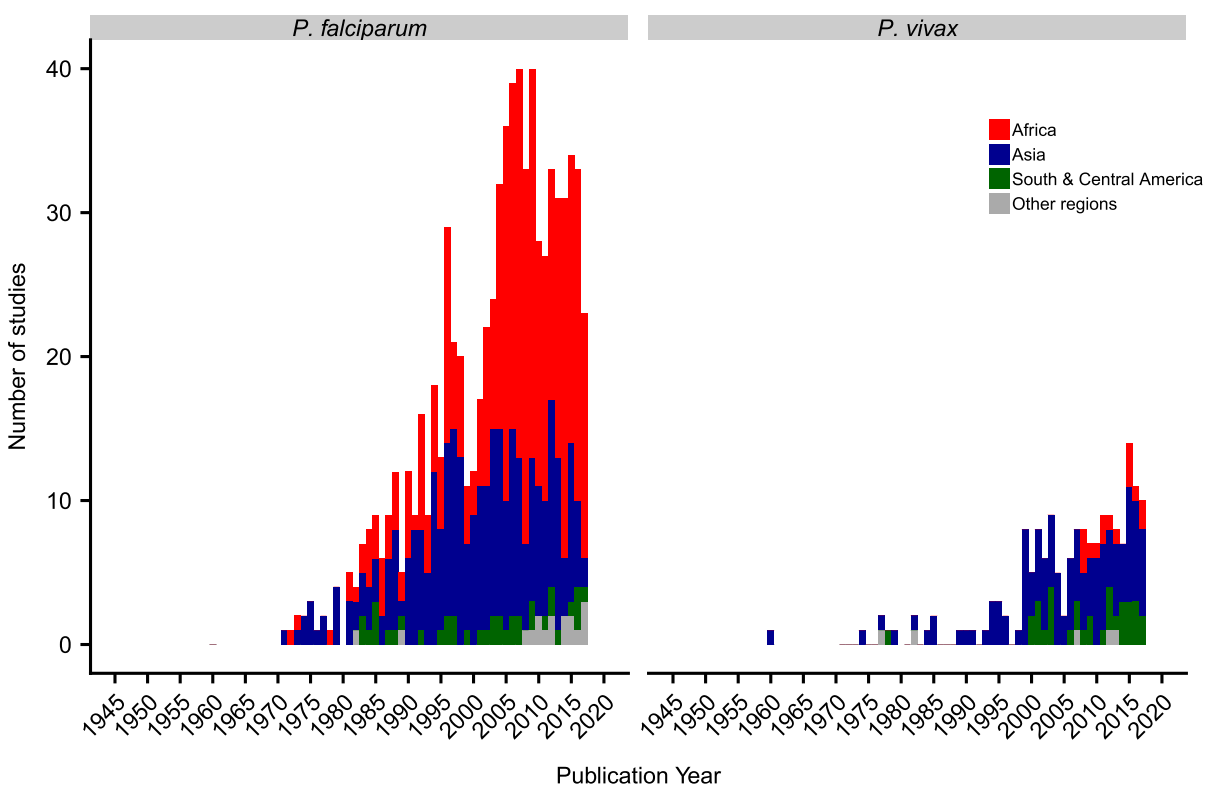

FIGURE 2. Number of publications per year by region. The year 2018 is not included as data were available for only part of the year. For clarity, the regions of Oceania and multi-region were classified as "other regions." This figure appears in color at www.ajtmh.org.

Malaria in returning travelers. Thirty-six studies investigated malaria in 4,652 returning travelers (Table 4), of which 20 (55.6\%) studies were conducted in Western Europe, mostly of travelers returning from sub-Saharan and Western Africa with $P$. falciparum. Twelve studies were undertaken in the United States, all of which enrolled returning military personnel with $P$. vivax most commonly from the Pacific $(n=5)$ or Vietnam $(n=3)$ between 1946 and 1997. Two studies were conducted in Australia and both included military personnel. Of the 75 treatment arms, artemisinin-based therapies were investigated in only four (5.3\%).

\section{DISCUSSION}

This study highlights the increasing volume of research on antimalarial therapy conducted across the world since the end of World War II. Most studies in the previous century were conducted in Asia, especially in Thailand, but these have declined in recent decades along with the marked reduction of $P$. falciparum malaria in the region. At the same time, there has been a substantial rise in contributions from research centers in sub-Saharan Africa, particularly in clinical trials assessing ACTs for the treatment of $P$. falciparum. According to the 2019 World Malaria Report, $93 \%$ of malaria cases

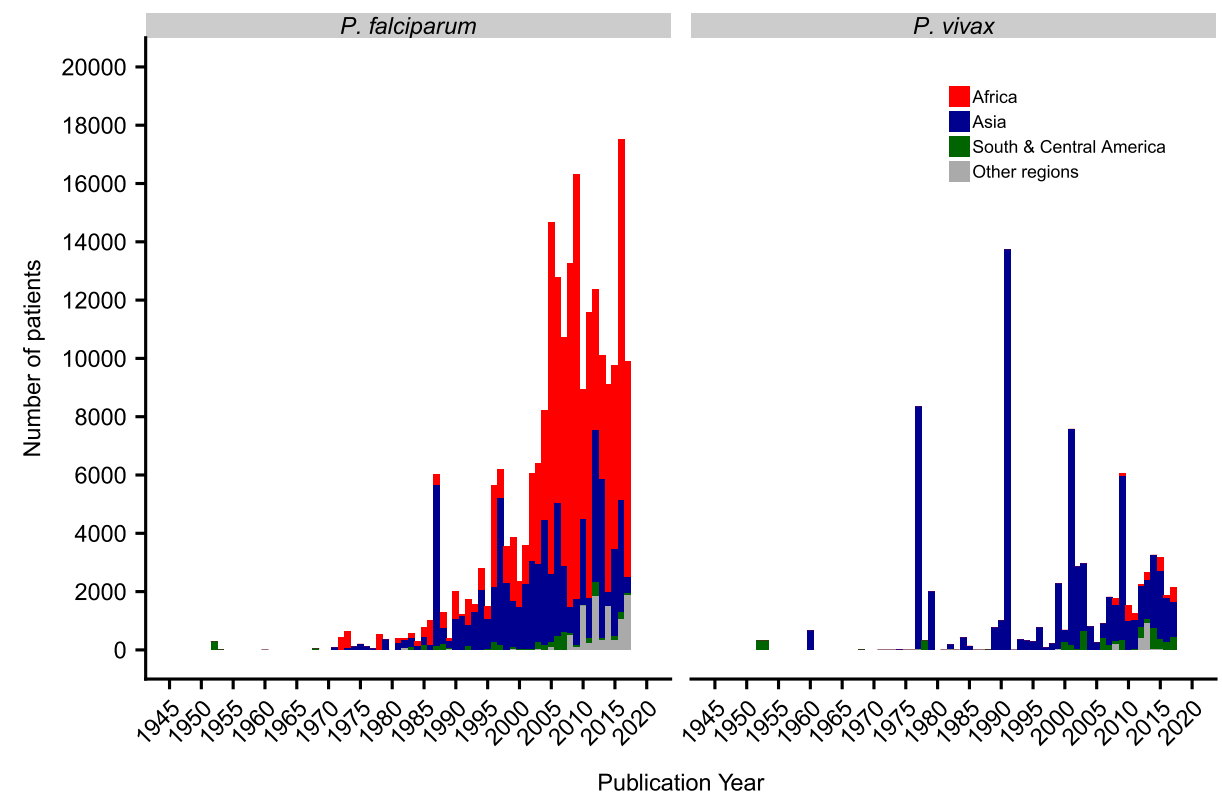

FIGURE 3. Number of patients recruited per year by region. The year 2018 is not included as data were available for only part of the year. For clarity, the regions of Oceania and multi-region were classified as "other regions." In Plasmodium falciparum studies, the spikes in 1987 and 1997 are due to large studies conducted at the Thai-Myanmar border. In P. vivax studies, the spikes in 1977, 1991, 2001, and 2009 were all relapse studies. This figure appears in color at www.ajtmh.org. 
A

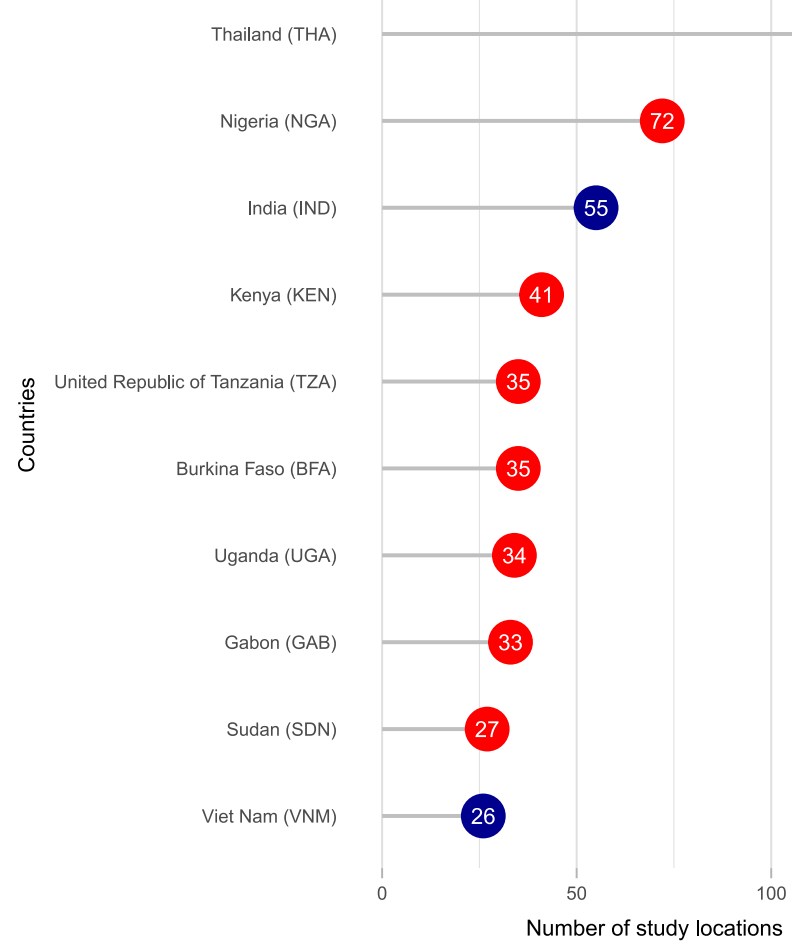

B

P. vivax

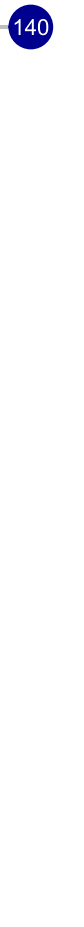

FIGURE 4. Most common study locations. (A) Plasmodium falciparum. (B) Plasmodium vivax. Numbers indicate the number of studies that were located in that country, including multicenter studies. This figure appears in color at www.ajtmh.org.

occur in the African region, with six countries contributing more than half of all cases worldwide -Nigeria, the Democratic Republic of the Congo, Uganda, Côte d'Ivoire, Mozambique, and Niger. ${ }^{15}$ These countries constituted $17.4 \%$ (54/310) of study locations in the last 5 years since 2013 (Supplemental Figure 1), suggesting a reasonable volume of research activity in the most affected countries. Yet, other heavily affected countries such as Rwanda, Guinea, or Central African Republic had very few studies published in the last 5 years compared with, for example, Kenya, which may reflect the distribution of the most active research groups globally. Because of the heterogeneous nature of malaria transmission and resistance, the provision of efficacy data that is spatially and

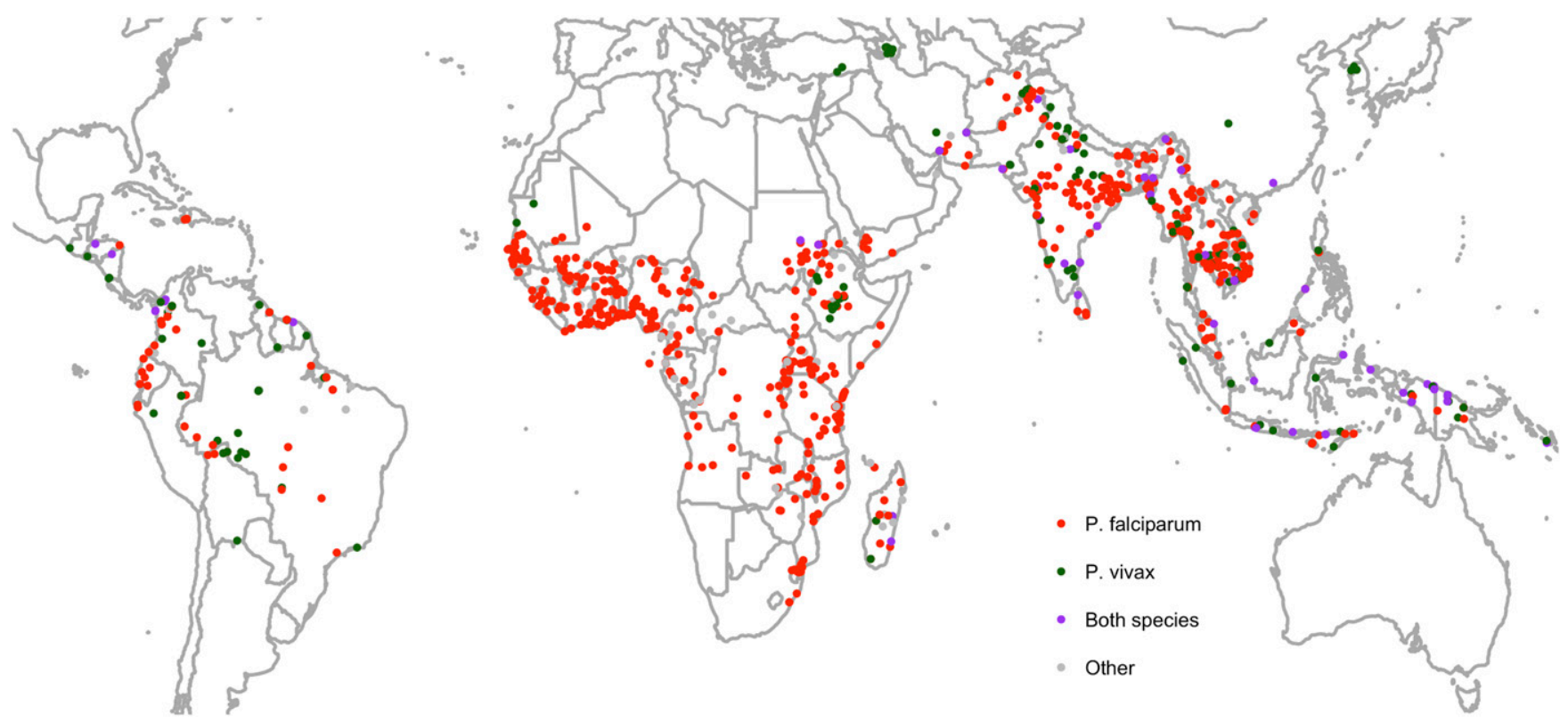

FIGURE 5. Distribution of study sites. Each dot represents a study site. North America, Europe, and the far north and south have been cropped for clarity as there were no study sites in these areas. This figure appears in color at www.ajtmh.org. 
A

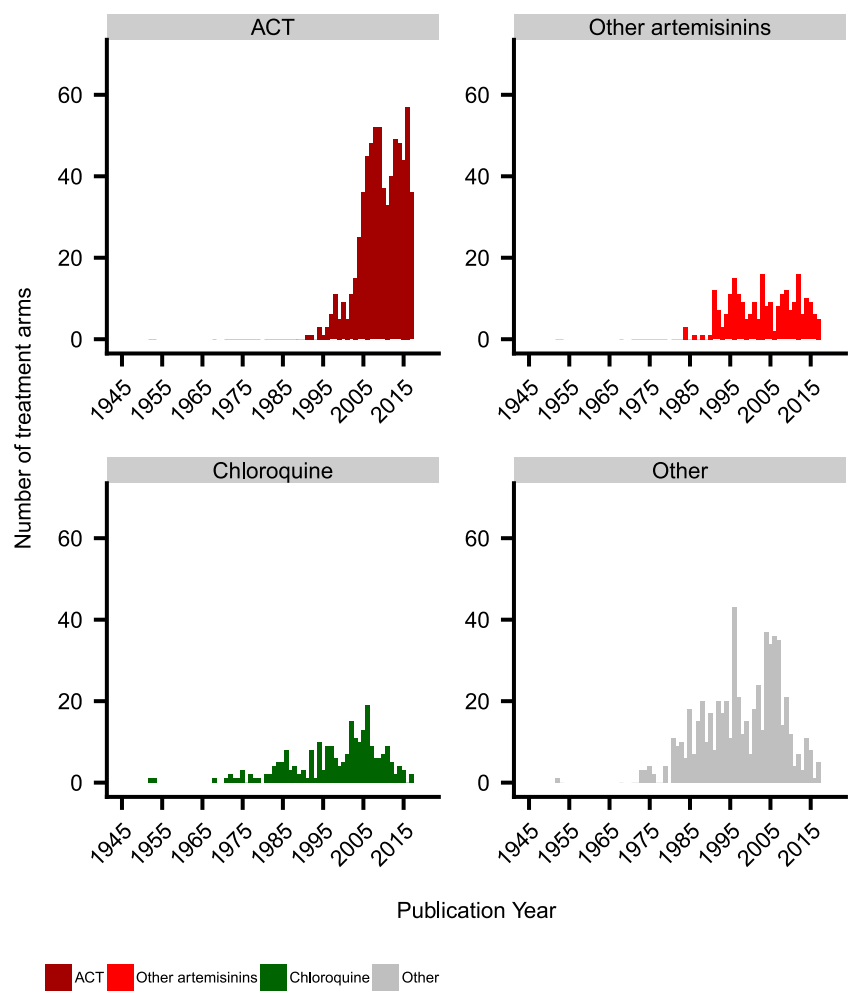

B

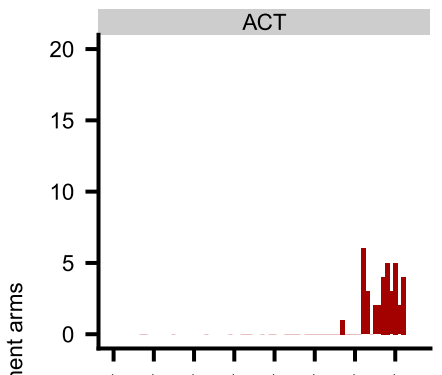

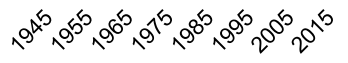

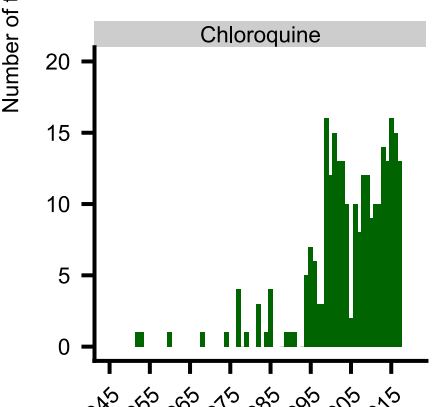

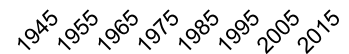

P. vivax

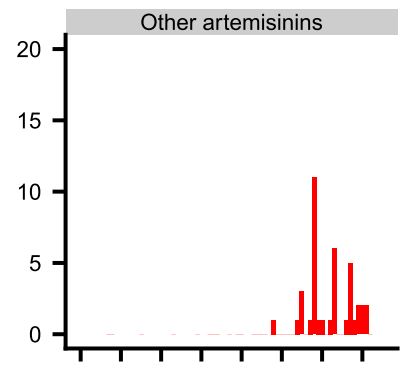

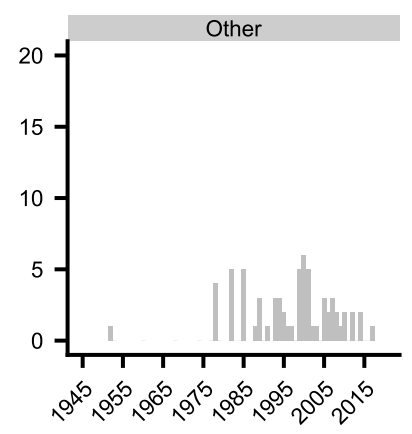

Publication Year

FIGURE 6. Number of treatment arms per year by drug type. (A) Plasmodium falciparum. (B) Plasmodium vivax. The year 2018 is not included as data were available for only part of the year. For clarity, chloroquine in combination with other drugs was included with "chloroquine," and all quinine combinations were combined with "other."This figure appears in color at www.ajtmh.org.

temporally specific is crucial for enabling policy-makers to optimize localized antimalarial policy.

Many trials conducted by national malaria control programs as part of routine surveillance for antimalarial resistance often remain unpublished and unavailable to the scientific community. For those studies which are published, the delay between the end of participant recruitment and publication is often long, with a median of 2 years and a maximum of 13 years in our study, highlighting the continuous challenge of publishing results in a timely manner that can provide policymakers with contemporaneous data. In the era of increasing resistance of $P$. falciparum to artemisinin and partner drugs, and $P$. vivax to chloroquine, novel ways of reporting and sharing unpublished trial data need to be explored so that the antimalarial resistance landscape can be kept up to date.

There has been a marked rise in the number of studies assessing $P$. vivax, reflecting a growing recognition of the burden of this parasite in Asia, the Americas, and the Horn of Africa. In particular, the recent interest in testing ACTs for $P$. vivax is a reflection of the need to find alternative treatments against chloroquine-resistant parasites and highlights the benefits of developing tools such as the Vivax Surveyor to monitor resistance and identify gaps in our knowledge for this historically less well studied species. ${ }^{2,16}$ Notably, only 23 studies assessed the efficacy of antimalarials in nonfalciparum or non-vivax species (either alone or alongside $P$. falciparum or $P$. vivax), indicating the limited evidence that currently exists for their treatment.

Children younger than 5 years are particularly vulnerable to malaria, accounting for $67 \%$ of malaria deaths worldwide in
2018 , and accordingly, $57 \%$ of studies in the database included this age-group. ${ }^{15}$ Since the 2000 s, there have also been more studies (mainly $P$. falciparum in Africa) that focus specifically on children younger than 5 years. Pregnant women, a similarly vulnerable group, represented just $5 \%$ of the total studies published, highlighting a marked lack of evidence in this population. ${ }^{17}$

Finally, whereas some treatments such as ACTs have been studied extensively, some compounds or combinations have been hardly studied. A collation of the current knowledge can, therefore, be used to avoid unnecessary duplication of future effort and provide an easily accessible way to analyze knowledge gaps to guide directions for further research.

Our study has several limitations. The WWARN library is the culmination of iterative refinements since 2011, and so there have inevitably been some discrepancies in methods that would not be the case if the library was created de novo today. It is possible that some studies were missed, especially those published in regional journals or foreign language articles that could not be fully accessed and/or extracted. However, the resources required for such an effort would add comparably little value to the existing resource assembled here. By excluding studies with less than 28 days of follow-up, we have also excluded proportionally more of the earlier published studies than the more recent, which could have contributed to the temporal trends described.

High-quality systematic reviews and meta-analyses are regarded as the highest level in the hierarchy of evidencebased medicine and are used to inform health policy. In practice, conducting reviews that uphold methodological 
A

A

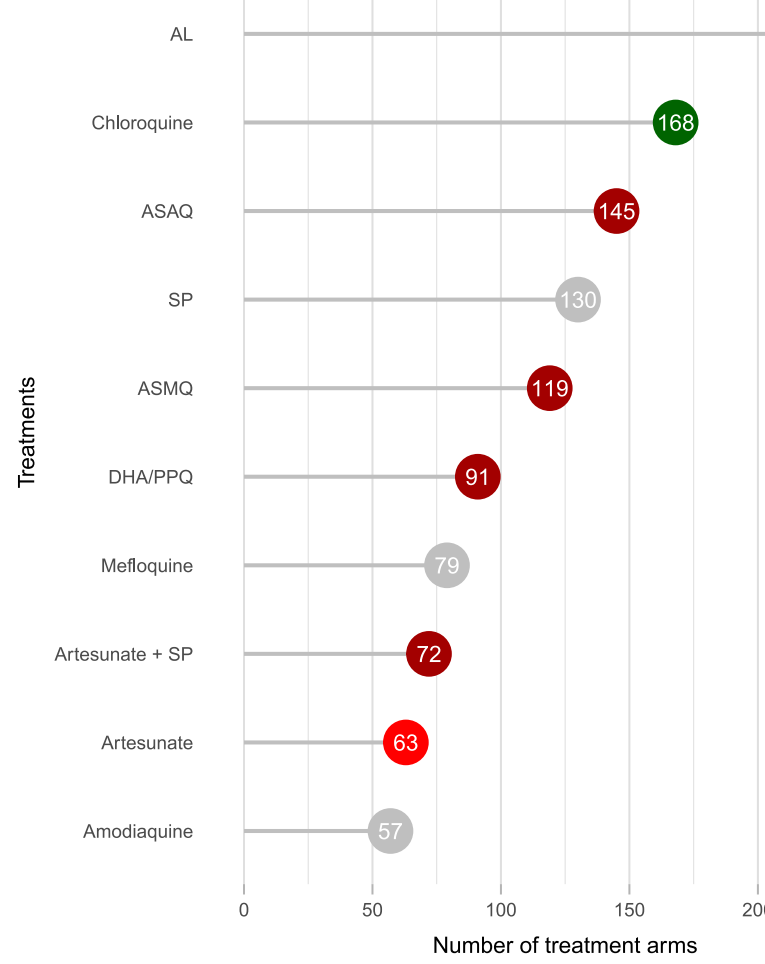

B

B $\quad$ P. vivax

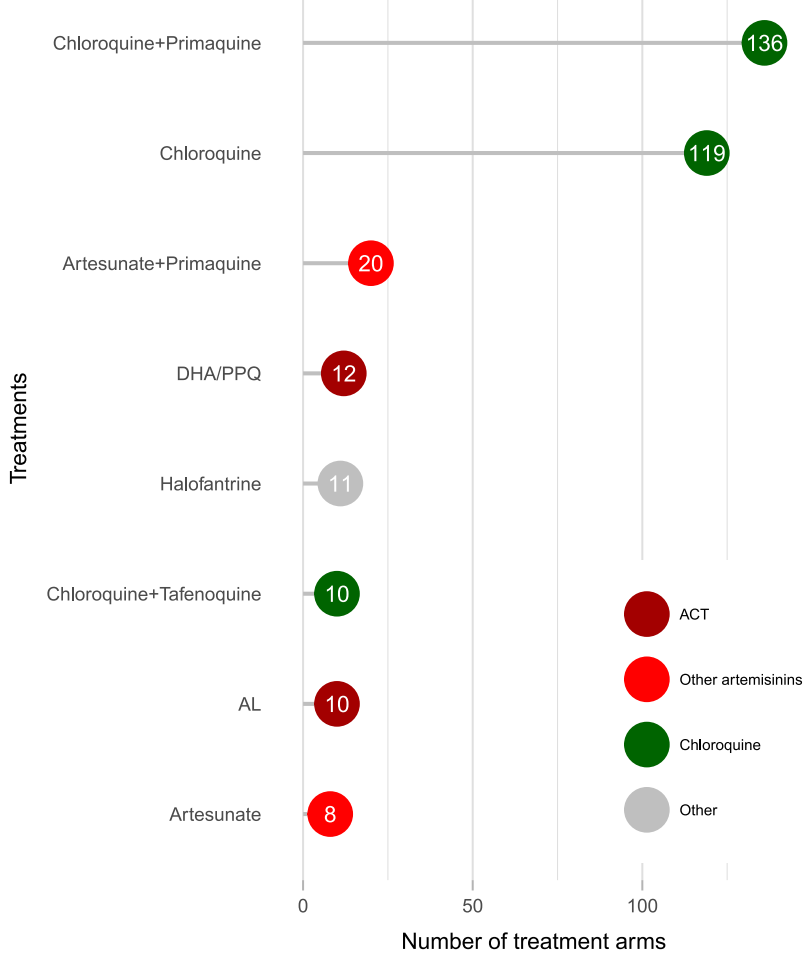

FiguRE 7. Most common treatment regimens by number of treatment arms. (A) Plasmodium falciparum. (B) Plasmodium vivax. Numbers indicate the number of treatment arms that assessed the drug. For clarity, chloroquine in combination with other drugs was included with "chloroquine," and all quinine combinations were combined with "other." $\mathrm{AL}=$ artemether-lumefantrine; $\mathrm{ASAQ}=$ artesunate-amodiaquine; $\mathrm{ASMQ}=$ artesunatemefloquine; $\mathrm{DHA} / \mathrm{PPQ}=$ dihydroartemisinin-piperaquine; $\mathrm{SP}=$ sulfadoxine-pyrimethamine. This figure appears in color at www.ajtmh.org.

rigor while simultaneously using contemporary knowledge is extremely challenging. A study by Shojania et al. estimated that $7 \%$ of systematic reviews fail to incorporate the latest evidence on the day that they are published, and this figure rises to $23 \% 2$ years after publication. ${ }^{18}$ In the rapidly changing field of malaria, the high rates of production of new evidence often exceed the speed with which evidence is synthesized, thus hindering the dissemination of knowledge and translation of knowledge into practice. ${ }^{19}$

In recent years, there have been several innovations that aim to bridge this gap. Systematically constructed, standardized databases can improve efficiency and completeness of systematic reviews, and incorporation of individual patient data allow even greater power to gain new insights. ${ }^{20,21}$ Another emerging concept is that of "living" systematic reviews, which use simplified methods to regularly update reviews and facilitate downstream products such as living guidelines. ${ }^{22}$

The WWARN clinical trials publication library can be used as an upstream contributor to this evolving evidence synthesis landscape, a contemporaneous database of publications, which can be explored easily to understand the extent and nature of knowledge. It will serve as an inventory to inform the availability of data for secondary use, aligning with recent recommendations from policy-makers and scientific journals to support a policy of data sharing. ${ }^{23-26}$ Similar endeavors, termed evidence mapping, have been conducted in other fields, but to our knowledge, this library is the first of its kind in the field of global infectious diseases. ${ }^{27,28}$

Going forward, the library will be updated every 6 months using a search strategy ratified by a systematic review search expert.
Results will be screened in specialist software by two independent reviewers, and data extracted by one reviewer and checked by another reviewer. Each update will be recorded in full to enable reporting according to Preferred Reporting Items for Systematic Reviews and Meta-Analyses (PRISMA) guidelines. The standard operating procedure, detailing the search strategy, screening, and extraction methods, will be regularly modified to ensure it captures all relevant publications such as those studying novel antimalarial drugs. With each update, the most recent version of the library with key extracted variables will be made open access on the WWARN website at https:// www.wwarn.org/tools-resources/literature-reviews/wwarnclinical-trials-publication-library. All users will be able to filter and view key variables of relevant studies. The organization of the library will be under continual review, to ensure that it remains easily usable and meets changing needs. It is planned that it will also use novel data visualizations similar to the current WWARN Vivax Surveyor. ${ }^{16}$

Lessons learnt from the WWARN experience are being applied and adapted for other disease themes within the Infectious Diseases Data Observatory, the umbrella platform of WWARN. Search strategies used in previously published systematic reviews on schistosomiasis, soil-transmitted helminths, and visceral leishmaniasis, along with a current review on Chagas disease, will be revised, and an up-to-date search will be planned prospectively every 6 months. ${ }^{29-31}$ Clinical study libraries, similar to the WWARN clinical trials publication library, will be created for each of the aforementioned disease themes and made available as online open-access resources. 
A

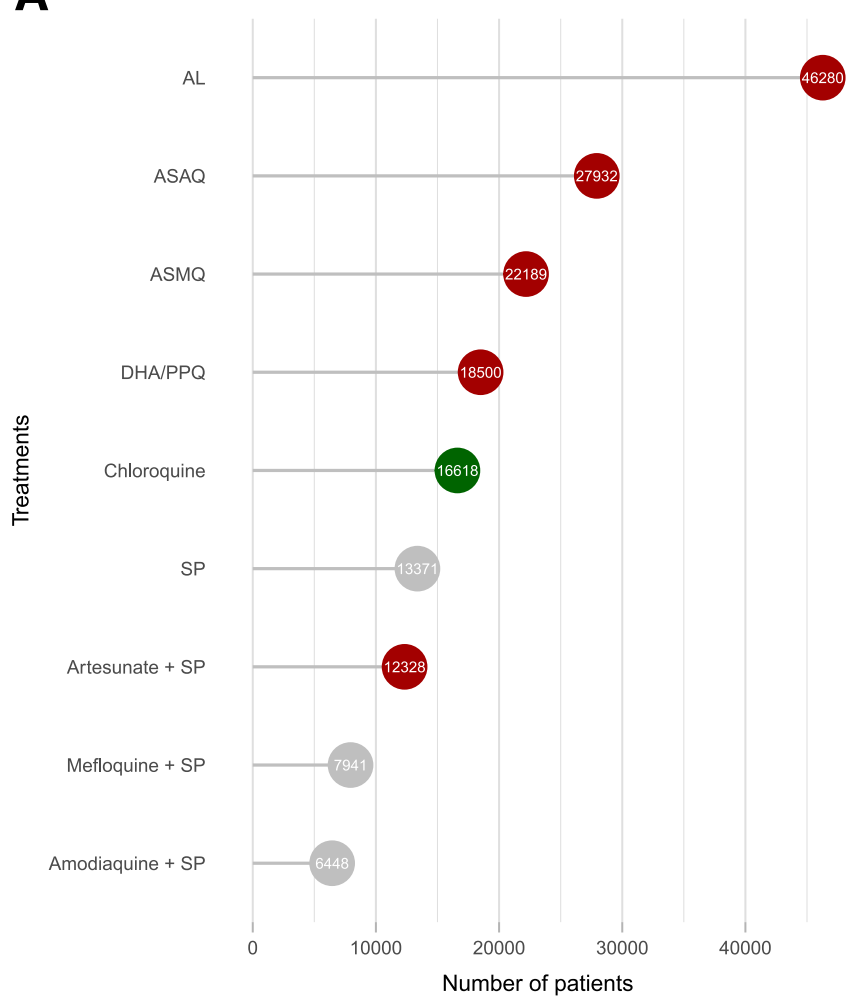

B P. vivax

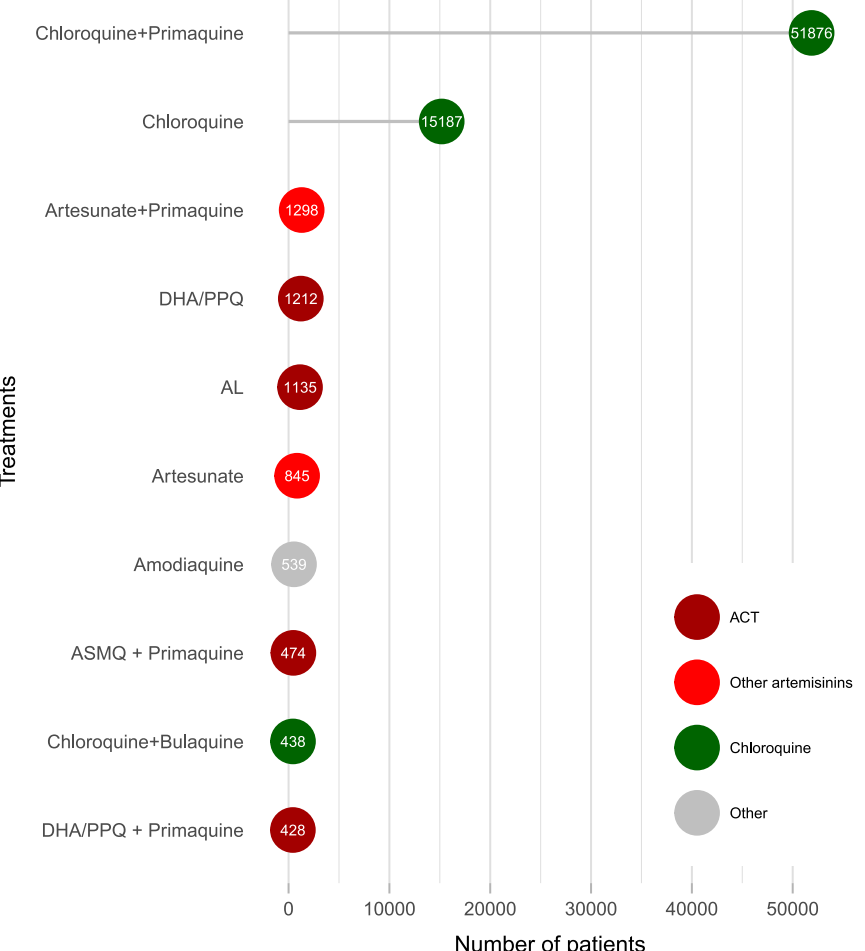

FIgURE 8. Most common treatment regimens by number of recruited patients. (A) Plasmodium falciparum. (B) Plasmodium vivax. Numbers indicate the number of patients who received the drug. For clarity, chloroquine in combination with other drugs was included with "chloroquine," and all quinine combinations were combined with "other." $\mathrm{AL}=$ artemether-lumefantrine; $\mathrm{ASAQ}=$ artesunate-amodiaquine; $\mathrm{ASMQ}=\mathrm{artesunate-}$ mefloquine; $\mathrm{DHA} / \mathrm{PPQ}=$ dihydroartemisinin-piperaquine; $\mathrm{SP}=$ sulfadoxine-pyrimethamine. This figure appears in color at www.ajtmh.org.

\section{CONCLUSION}

In conclusion, this article summarizes the antimalarial efficacy trial landscape since the 1940s and provides a detailed resource for further analyses. The WWARN clinical trials publication library highlights the potential for a novel approach to evidence synthesis and dissemination that can be adapted for other global infectious diseases.

Received September 23, 2019. Accepted for publication March 14, 2020.

Published online May 18, 2020.

TABLE 4

Summary of studies in returning travelers

\begin{tabular}{|c|c|c|c|c|c|c|}
\hline \multirow[b]{2}{*}{ Region } & \multirow[b]{2}{*}{ Published year(s) } & \multicolumn{4}{|c|}{ Species } & \multirow[b]{2}{*}{ Treatment regimens $\dagger$} \\
\hline & & $\begin{array}{l}\text { Plasmodium } \\
\text { falciparum }\end{array}$ & $\begin{array}{c}\text { Plasmodium } \\
\text { vivax }\end{array}$ & Both & $\mathrm{N} / \mathrm{A}^{*}$ & \\
\hline Europe $(n=20) \ddagger$ & 1970-2012 & 13 & 1 & 2 & 4 & $\begin{array}{l}\text { Quinine combinations (8) } \\
\text { Mefloquine (8) } \\
\text { Halofantrine (7) } \\
\text { Artemether combinations (4) } \\
\text { Atovaquone combinations (5) } \\
\text { Chloroquine combinations (2) }\end{array}$ \\
\hline United States $(n=12)$ & $1946-1997$ & 0 & 11 & 1 & 0 & $\begin{array}{l}\text { Chloroquine combinations (17) } \\
\text { Quinine combinations (9) } \\
\text { Quinacrine combinations (4) } \\
\text { Primaquine (1) }\end{array}$ \\
\hline Japan $(n=2)$ & 1986 and 1987 & 0 & 0 & 1 & 1 & $\begin{array}{l}\text { Chloroquine (2) } \\
\text { Quinine (1) } \\
\text { Sulfadoxine-pyrimethamine (2) } \\
\text { Sulfamonomethoxine-pyrimethamine (2) }\end{array}$ \\
\hline Australia $(n=2)$ & 1948 and 2007 & 1 & 1 & 0 & 0 & $\begin{array}{l}\text { Quinacrine (1) } \\
\text { Tafenoquine (1) } \\
\text { Quinine (1) }\end{array}$ \\
\hline
\end{tabular}

${ }^{\star} \mathrm{N} / \mathrm{A}$ refers to studies in which species data were not specified.

$\dagger$ Five treatment regimens were unknown because of lack of full text.

$\ddagger$ These consisted of France (9), Germany (3), Denmark (2), Russia (1), United Kingdom (1), and Italy (1). Three were multi-Europe, one of which also included a center in Colombia. 
Note: Supplemental tables and figures appear at www.ajtmh.org.

Acknowledgments: We thank the librarians at the University of Oxford Bodleian Library; in particular, Eli Harriss who conducted and deduplicated the searches, and James Shaw who helped retrieve the fulltext articles.

Financial support: The WWARN is supported by the Bill \& Melinda Gates Foundation and the ExxonMobil Foundation. J. T. is supported by Oxford University Clinical Academic Graduate School, and during this work, P. S. and M. S. H. were supported by a Clinical Research and Development Fellowship scheme from TDR, the UNICEF/UNDP/ World Bank/WHO Special Programme for Research and Training in Tropical Diseases. R. J. C. is supported by a Postgraduate Australian National Health and Medical Research Council (NHMRC) Scholarship. R. N. P. is a Wellcome Trust senior fellow in Clinical Science (200909).

Authors' addresses: Junko Takata, Rebekah Burrow, Brittany Maguire, Georgina Humphreys, Debashish Das, and Philippe Guerin, Infectious Diseases Data Observatory (IDDO), Centre for Tropical Medicine and Global Health, University of Oxford, Oxford, United Kingdom, E-mails: junko.takata@ndm.ox.ac.uk, rebekah.burrow@wwarn.org, brittany. maguire@iddo.org, georginahumphreys@gmail.com, debashish.das @wwarn.org, and philippe.guerin@wwarn.org. Paul Sondo, Institut de Recherche en Sciences de la Santé (IRSS)/Clinical Research Unit of Nanoro (CRUN), Nanoro, Burkina Faso, E-mail: paul.sondo@ wwarn.org. Mohammad Sharif Hossain, International Centre for Diarrhoeal Disease Research, Bangladesh (icddr,b), Dhaka, Bangladesh. E-mail: sharif.hossain@wwarn.org. Robert J. Commons and Ric N. Price, Global Health Division, Menzies School of Health Research, Charles Darwin University, Darwin, Australia, E-mails: rob.commons@wwarn.org and ric.price@menzies.edu.au.

This is an open-access article distributed under the terms of the Creative Commons Attribution (CC-BY) License, which permits unrestricted use, distribution, and reproduction in any medium, provided the original author and source are credited.

\section{REFERENCES}

1. Ashley EA, Dhorda M, Fairhurst RM, Amaratunga $P$, Lim S, Suon $\mathrm{S}$, Anderson JM, 2014. Spread of artemisinin resistance in Plasmodium falciparum malaria. N Engl J Med 371: 411-423.

2. Commons RJ, Thriemer K, Humphreys G, Suay I, Sibley $\mathrm{CH}$, Guerin PJ, Price RN, 2017. The vivax surveyor: online mapping database for Plasmodium vivax clinical trials. Int $J$ Parasitol Drugs Drug Resist 7: 181-190.

3. Price RN, Seidlein von L, Valecha N, Nosten F, Baird KJ, White NJ, 2014. Global extent of chloroquine-resistant Plasmodium vivax: a systematic review and meta-analysis. Lancet Infect Dis 14: 982-991.

4. John G, Douglas NM, Seidlein von L, Nosten F, Baird KJ, White NJ, Price RN, 2012. Primaquine radical cure of Plasmodium vivax: a critical review of the literature. Malar J 11: 280.

5. Commons RJ, Simpson JA, Thriemer K, Hossain MS, Douglas NM, Humphreys GS, Sibley CH, Guerin PJ, Price RN, 2018. Risk of Plasmodium vivax parasitaemia after Plasmodium falciparum infection: a systematic review and meta-analysis. Lancet Infect Dis 19: 91-101.

6. WorldWide Antimalarial Resistance Network (MWARN) AL Dose Impact Study Group, 2015. The effect of dose on the antimalarial efficacy of artemether-lumefantrine: a systematic review and pooled analysis of individual patient data. Lancet Infect Dis 15: 692-702.

7. The WorldWide Antimalarial Resistance Network (WWARN), 2015. The effect of dosing strategies on the therapeutic efficacy of artesunate-amodiaquine for uncomplicated malaria: a metaanalysis of individual patient data. BMC Med 13: S8-S19.

8. The WorldWide Antimalarial Resistance Network (WWARN) DP Study Group, 2013. The effect of dosing regimens on the antimalarial efficacy of dihydroartemisinin-piperaquine: a pooled analysis of individual patient data. PLos Med 10: e1001564.

9. Das D, Price RN, Bethell D, Guerin PJ, Stepniewska K, 2013. Early parasitological response following artemisinin-containing regimens: a critical review of the literature. Malar J 12: 1-13.

10. WWARN Clinical Trials Publication Library, 2020. Available at: https://www.wwarn.org/tools-resources/literature-reviews/ wwarn-clinical-trials-publication-library. Accessed April 7, 2020.

11. World Health Organization, 2009. Methods for Surveillance of Antimalarial Drug Efficacy. Geneva, Switzerland: WHO Press.

12. Harris PA, Taylor R, Thielke R, Payne J, Gonzalez N, Conde JG, 2009. Research electronic data capture (REDCap) - a metadata-driven methodology and workflow process for providing translational research informatics support. J Biomed Inform 42: 377-381.

13. Harris PA et al.; The REDCap Consortium, 2019. The REDCap consortium: building an international community of software platform partners. J Biomed Inform 95: 103208.

14. United Nations, 1970. Standard Country or Area Codes for Statistical Use (M49). Available at: https://unstats.un.org/unsd/methodology/ m49/overview/. Accessed July 31, 2019.

15. World Health Organization, 2019. World Malaria Report 2019. Geneva, Switzerland: World Health Organization. 1-232.

16. The WorldWide Antimalarial Resistance Network (WWARN), 2016. The Vivax Surveyor. Available at: https://www.wwarn.org/ tracking-resistance/vivax-surveyor. Accessed July 31, 2019.

17. Saito M, Gilder ME, Nosten F, McGready R, Guérin PJ, 2017. Systematic literature review and meta-analysis of the efficacy of artemisinin-based and quinine-based treatments for uncomplicated falciparum malaria in pregnancy: methodological challenges. Malar J 16: 488.

18. Shojania KG, Sampson M, Ansari MT, Ji J, Doucette S, Moher D, 2007. How quickly do systematic reviews go out of date? A survival analysis. Ann Intern Med 147: 224-233.

19. World Health Organization, 2006. Bridging the "Know-Do" Gap. Available at: https://www.measureevaluation.org/resources/ training/capacity-building-resources/high-impact-researchtraining-curricula/bridging-the-know-do-gap.pdf. Accessed July $31,2019$.

20. Neville $\mathrm{J}$ et al., 2015. Development of a unified clinical trial database for Alzheimer's disease. Alzheimer's Demen 11: 1212-1221.

21. Humphreys GS, Tinto $\mathrm{H}$, Barnes KI, 2019. Strength in numbers: the WWARN case study of purpose-driven data sharing. Am J Trop Med Hyg 100: 13-15.

22. Elliott JH, Turner T, Clavisi O, Higgins JP, Mavergames C, Gruen $\mathrm{RL}, 2014$. Living systematic reviews: an emerging opportunity to narrow the evidence-practice gap. PLos Med 11: 1-6.

23. Ohmann $\mathrm{C}$ et al., 2017. Sharing and reuse of individual participant data from clinical trials: principles and recommendations. BMJ Open 7: e018647.

24. Mello MM, Francer JK, Wilenzick M, Teden P, Bierer BE, Barnes $M, 2013$. Preparing for responsible sharing of clinical trial data. N Engl J Med 369: 1651-1658.

25. Vickers $A, 2016$. Sharing raw data from clinical trials: what progress since we first asked "Whose data set is it anyway?" Trials 17: 227.

26. Institute of Medicine, 2015. Sharing Clinical Trial Data. Washington, DC: The National Academies Press, 1-304.

27. De Silva S, Bailey AP, Parker AG, Montague AE, Hetrick SE, 2018. Open-access evidence database of controlled trials and systematic reviews in youth mental health. Early Interv Psychiatry 12: 474-477.

28. Bragge P, Clavisi O, Turner T, Tavender E, Collie A, Gruen RL, 2011. The global evidence mapping initiative: scoping research in broad topic areas. BMC Med Res Methodol 11: 92.

29. Julé AM, Vaillant M, Lang TA, Guérin PJ, Olliaro PL, 2016. The schistosomiasis clinical trials landscape: a systematic review of antischistosomal treatment efficacy studies and a case for sharing individual participant-level data (IPD). PLoS Negl Trop Dis 10: e0004784.

30. Halder JB, Benton J, Julé AM, Guérin PJ, Olliaro PL, Basáñez MG, Walker M, 2017. Systematic review of studies generating individual participant data on the efficacy of drugs for treating soil-transmitted helminthiases and the case for data-sharing. PLoS Negl Trop Dis 11: e0006053.

31. Bush JT, Wasunna M, Alves F, Alvar J, Olliaro PL, Otieno M, Sibley $\mathrm{CH}$, Strub Wourgaft N, Guérin PJ, 2017. Systematic review of clinical trials assessing the therapeutic efficacy of visceral leishmaniasis treatments: a first step to assess the feasibility of establishing an individual patient data sharing platform. PLoS Negl Trop Dis 11: e0005781. 